\title{
Alginate-Chitosan Particulate Delivery Systems for Mucosal Immunization against Tuberculosis
}

\author{
Liliana Aranha Caetano ${ }^{1,2}$, Lara Figueiredo ${ }^{1}$, António J. Almeida ${ }^{1}$, Lídia M. D. Gonçalves ${ }^{1}$ \\ ${ }^{I}$ Faculdade de Farmácia, Universidade de Lisboa iMed.UL, Lisboa, Portugal; (lacaetano@ff.ul.pt) \\ ${ }^{2}$ Escola Superior de Tecnologia da Saúde de Lisboa, Instituto Politécnico de Lisboa. Lisboa, Portugal
}

\begin{abstract}
Although vaccination is still the most cost-effective strategy for tuberculosis control, there is an urgent need for an improved vaccine. Current BCG vaccine lacks efficacy in preventing adult pulmonary tuberculosis, the most prevalent form of the disease. Targeting nasal mucosa, Mycobacterium tuberculosis infection site, will allow a simpler, less prone to risk of infection and more effective immunization against disease. Due to its biodegradable, immunogenic and mucoadhesive properties, chitosan particulate delivery systems can act both as carrier and as adjuvant, improving the elicited immune response. In this study, BCG was encapsulated in alginate and chitosan microparticles, via a mild ionotropic gelation procedure with sodium tripolyphosphate as a counterion. The particulate system developed shows effective modulation of BCG surface physicochemical properties, suitable for mucosal immunization. Intracellular uptake was confirmed by effective transfection of human macrophage cell lines.
\end{abstract}

Index Terms-BCG, microencapsulation, chitosan, mucosal immunization

\section{INTRODUCTION}

Tuberculosis remains the leading cause of death from infectious diseases throughout the world, killing an estimated 1.9 million people every year. The only available vaccine - the attenuated Mycobacterium bovis Bacillus Calmette-Guerin (BCG) lacks efficacy in preventing adult pulmonary tuberculosis, the most prevalent form of the disease. Also, there is the risk of infection from HIV, hepatitis, and other serious diseases due to unsterilized needles during subcutaneous administration. [1]

Mycobacterium tuberculosis is usually transmitted via aerosols and establishes itself in the lungs. Thus, mucosal vaccination at this site can help to prevent pathogen entry and infection [2]. A vaccine with an appropriate adjuvant can induce both mucosal and systemic immune responses, preventing not only infectious disease but also colonization of mucosal surfaces [3].

Biodegradable particulate delivery systems have been successfully used in recent vaccine development. Not only particles present adjuvant properties, favoring antigen uptake by antigen presenting cells such as macrophages, they also act as antigen depot, increasing the immune response at the target site. Particle features, such as size and composition, can direct a different pattern of $\mathrm{MHC}$ presentation and acquired immunity, thus, improving the immune response [4-6]. The encapsulation of live attenuated BCG for storage extension has already been described [7], and mucosal delivery systems designed to enhance the immune response following mucosal immunization have been evaluated for efficacy in tuberculosis vaccines [8].

\section{GOALS}

In this work we developed a cell-based particulate delivery system, using alginate-chitosan microparticles to encapsulate monodisperse BCG. The cell viability under different conditions was evaluated. The capacity to transfect human macrophage cell lines was also studied.

\section{MATERIALS AND METHODS}

\section{A. Materials}

Chitosan of different molecular weight (low molecular weight (CH-LMW, $150 \mathrm{kDa})$; medium molecular weight $(\mathrm{CHMMW}), \mathrm{MW}=450 \mathrm{kDa})$ and high molecular weight 600 $\mathrm{kDa}(\mathrm{CH}-\mathrm{HMW})$, sodium alginate with different viscosities and TPP were obtained from Sigma Aldrich, UK. BCG strains (Pasteur and GFP) were kindly supplied by Prof. Elsa Anes (FFUL). All cell culture reagents were from Invitrogen, UK.

\section{B. Microparticles Preparation}

Alginate-chitosan microparticles containing whole live attenuated BCG were formulated avoiding solvents, high shear stress and high temperature. Microparticle formation was carried out via ionotropic gelation with sodium tripolyphosphate (TPP) [9]. Briefly, $2.0 \mathrm{~mL}$ of $0.1 \%$ TPP was added to $5.0 \mathrm{~mL}$ of $0.1 \%$ Chitosan containing different amounts of sodium alginate in water. The particles with BCG were prepared by adding different amounts of BCG to the solution of TPP containing chitosan and then alginate was added to this solution with agitation. 


\section{Characterization of Alginate/Chitosan microparticles}

The microparticles were assessed according to size distribution and surface charge (zeta potential) by dynamic light scattering and electrophoretic mobility, respectively (Malvern Instruments, UK). For particle size analysis, each sample was diluted to the appropriate concentration with filtered purified water. Each analysis was carried out in triplicate at $25^{\circ} \mathrm{C}$. For the determination of the electrophoretic mobility, samples were diluted with filtered purified water to an appropriate concentration to yield count rate per second (KCps) in the appropriate range. All measurements were performed in triplicate.

\section{Human Macrophages Cell Lines}

Different mycobacteria strains (M. bovis BCG and recombinant $M$. bovis BCG expressing Green Fluorescent Protein (GFP)) were tested in human macrophages THP1. Following the trypan blue exclusion method, macrophage live cells were differentiated with retinoic acid and maintained in Roswell Park Memorial Institute 1640 medium supplemented with $10 \%$ foetal bovine serum, 100-units $/ \mathrm{ml}$ penicillin, 100$\mu \mathrm{g} / \mathrm{ml}$ streptomycin and L-glutamine, in humidified atmosphere of $5 \% \quad \mathrm{CO}_{2}$ at $37^{\circ} \mathrm{C}$. BCG-beads intracellular uptake was monitored after infection of THP-1 cells, following colony- forming units (cfu).

\section{E. Cell Viability and Citotoxicity Assays}

BCG surface charge was modulated either through direct ressuspension in biocompatible media $(0,9 \% \mathrm{NaCl} /$ $0,025 \% \mathrm{CS}$ ), or via encapsulation in ALG/CS-TPP microparticles (M13 / H13). A micro colony-forming units assay was used during procedures, by counting bacteria able to produce colonies in a solid agar Middlebrook $7 \mathrm{H} 10$ medium supplemented with OADC, widely used to cultivate and access the CFUs in the specific case of fastidious slow growing mycobacteria such as M. Bovis [10].

Cell viability of BCG-beads in suspension was monitored through an assay using THP-1 cell line. A viability assay was performed using the common propidium iodide PI/SYBR 14 assay to assess viability of THP-1 cells in the presence of Alg:CS microparticles. Cells were incubated at $37^{\circ} \mathrm{C}$ for $72 \mathrm{~h}$.

\section{RESULTS AND DISCUSSION}

\section{A. Microparticles Preparation and Characterization}

Alginate was found to be a stabilizing agent to the produced microparticles under the used conditions. The size range was 12.5-16.3 $\mu \mathrm{m}$ (Fig. 1) and zeta potential varied from -14.9 to $-49.8 \mathrm{mV}$ for microparticles produced with low or medium viscosity alginate:low or medium molecular weight Chitosan:TPP (w:w:w) from 0.8:1:0.4 to 1:1:0.4.

The micrographs shown in Fig. 2 indicate the microparticles have a spherical shape and form homogeneous populations, which is consistent with previous described systems. No significant difference is observed for microparticles with and without encapsulated BCG.

\begin{tabular}{lrrrr}
\hline $\begin{array}{c}\text { ALG:CS:TPP ratio } \\
\text { (w:w:w) }\end{array}$ & $\mathbf{d}(\mathbf{0 , 1})$ & $\mathbf{d}(\mathbf{0 , 5})$ & $\mathbf{d}(\mathbf{0}, \mathbf{9})$ & Span * \\
\hline \multirow{2}{*}{$\begin{array}{l}\text { 1:1:0.4 } \\
\text { 0.8:1:0.4 }\end{array}$} & 5.450 & 12.520 & 28.047 & 1.805 \\
& 6,430 & 16,322 & 38,368 & 1,957 \\
\hline
\end{tabular}

* Span $=[d(0,9)-d(0,1)] / d(0,5)$

Fig. 1 - Mean Particle size of Alg/CS-TPP microparticles prepared with different proportions of alginate of Low and Medium viscosity and chitosan of Low and Medium Molecular weight (mean $\pm \mathrm{sd} ; \mathrm{n}=3$ ).

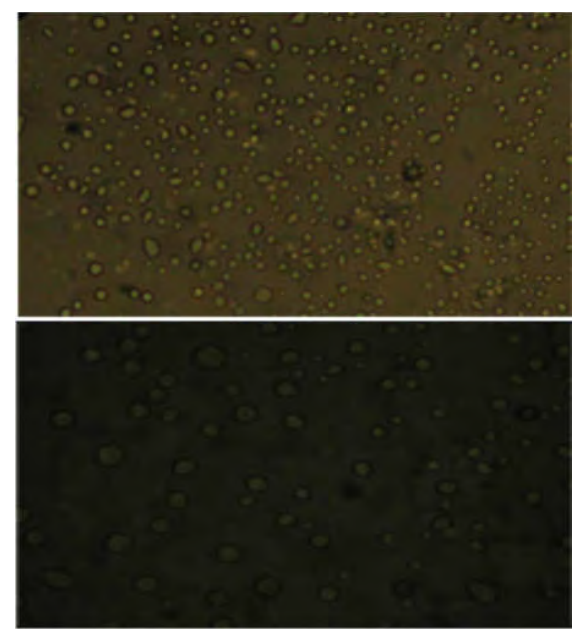

Fig. 2 - Contrast microphotographs showing alginate-chitosan microparticles (Alg/CS (w:w) 0.8:1).

\section{B. Cell viability and Cell transfection}

Microparticles containing encapsulated BCG were able to transfect THP-1 macrophage cell lines (Fig. 3). Cell viability measured by MTT reduction assay show that both microparticles with encapsulated BCG and BCG only, modulated by $\mathrm{Nacl} 0.9 \%$ or chitosan, present low toxicity levels (Fig.4), which is in agreement with the biocompatibility of the two polymers. 

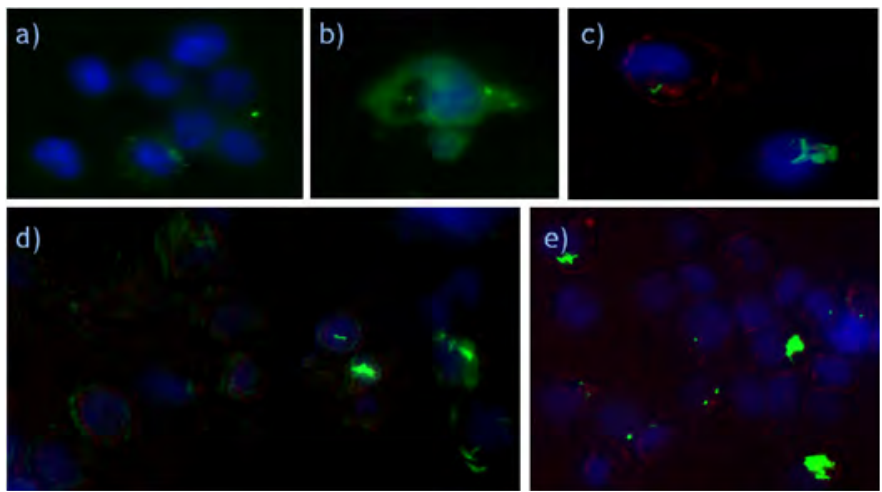

Fig. 3 - Fluorescence micrographs showing the intracellular uptake of particulated or modulated BCG-GFP. Blue: DAPI; Red: Lysotrack ${ }^{\mathrm{TM}}$; Green: GFP (Green fluorescence protein). Multiplicity of Infection: MOI $=\mathrm{n}$ BCG-GFP cells $/ \mathrm{n}$ THP-1 cells. A- M13_BCG (MOI of 1); B- $0,9 \% \mathrm{NaCl} B \mathrm{BCG}$ (MOI of 1); C- $0,025 \% \mathrm{CS}$ BCG (MOI of 10); D- $0,025 \% \mathrm{CS}$ BCG (MOI of 10 ); $\mathrm{E}-0, \overline{9 \%} \mathrm{NaCl} \mathrm{BCG}$ (MOI of 10 ).

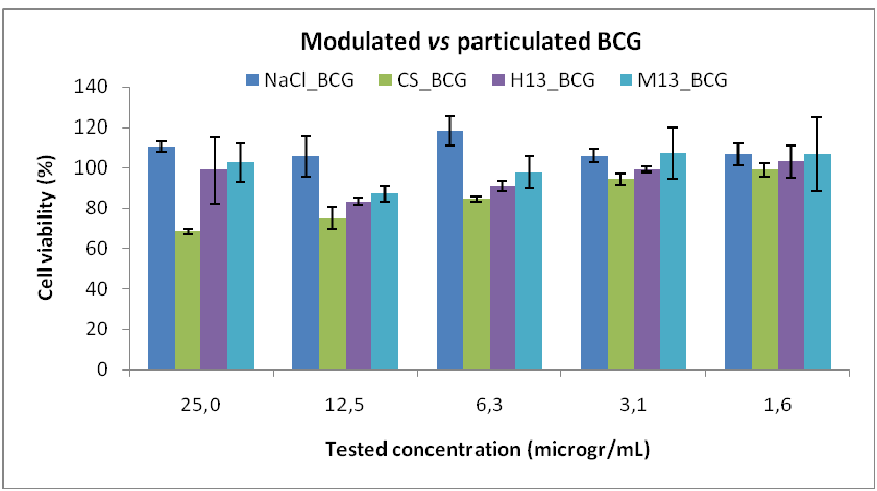

Fig. 4 - Cell viability of THP-1 cells transfected with Alg:CS microparticles of different proportions (w:w) containing encapsulated or modulated BCG-GFP, for several tested concentrations.

\section{CONCLUSIONS}

Present work proposes a new strategy with particulate delivery systems to design an improved anti-TB vaccine, capable of elicit a strong pulmonary mucosal response and to induce protective cell-mediated immunity, in a more stable and long-lasting formulation.

Preliminary results showed to be possible to modulate the surface physicochemical properties of BCG through coating/encapsulation of whole mycobacteria in suitable polymeric particulate carriers with immunological adjuvant properties.

\section{PlanNed DEVELOPMENTS}

Current work includes evaluation of the intracellular traffic of these beads to the phagolysosome compartment. Next steps will be the assessment of cell viability and formulation stability after freeze-drying and further testing of the innovative formulation as potential vaccine in mice, through a mycobacterial growth inhibition in vitro assay.

\section{ACKNOWLEDGMENTS}

The authors would like to thank to Prof. Elsa Anes (FFUL) and her team (Dr. David Pires, Dr. Nuno Carmo) by supplying strains (Pasteur and GFP), for access to the laboratory as well as for all the advice and practical support. This work was supported by Fundação para a Ciência e Tecnologia (Portugal) (PEst-OE/SAU/UI4013/2011).

\section{REFERENCES}

[1] Andersen P (2007) Vaccine strategies against latent tuberculosis infection. Trends Microbiol 15: 7-13.

[2] Doherty TM, Olsen AW, van Pinxteren L \& Adersen P (2002) Oral vaccination with subunit vaccines protects animals against aerosol infection withMycobacterium tuberculosis. Infect Immun 70: 31113121 .

[3] Bivas-Benita $M$, van Meijgaarden KE, Franken KL, Junginger HE, Borchard G, Ottenhoff TH \& Geluk A (2004) Pulmonary delivery of chitosan-DNA nanoparticles enhances the immunogenicity of a DNAvaccine encoding HLA-A 0201- restricted T-cell epitopes ofMycobacterium tuberculosis. Vaccine 22: 1609-1615.

[4] Storni T, Kondig TM, Senti G, Johansen P: Immunity in response to particulate antigen delivery systems. Adv Drug Deliv Rev 2005, 57:333355

[5] Johansen P, Storni T, Rettig L, Qiu Z, Der-Sarkissian A, Smith KA, Manolova V, Lang KS, Senti G, MullhauptBet al.: Antigen kinetics determines immune reactivity. Proc Natl Acad Sci U S A 2008, 105:5189-5194

[6] Xiang SD, Scholzen A, Minigo G, David C, Apostolopoulos V, Mottram PL, Plebanski M: Pathogen recognition and development of particulate vaccines: does size matter? Methods 2006, 40:1-9.

[7] Esquisabel A, Hernandez RM, Igartua M, Gascon AR, Calvo B, Pedraz JL: Preparation and stability of agarosemicrocapsules containing BCG. J Microencapsul 2002, 19:237-244.

[8] Davis SS (2001) Nasal vaccines. Adv Drug Deliver Rev 51: 21-42. de Souza Silva BD, da Silvia EB, do Nascimento IP, Guerreiro dos Reis $\mathrm{MC}$, Kipnis A \& Junqueira-Kipnis AP (2009) MPT-51/CpG DNAvaccine protects mice againstMycobacterium tuberculosis. Vaccine 27: 4402-4407.

[9] Calvo, P., Remuñán-López, C., Vila-Jato, J.L., Alonso, M.J. (1997). Novel hydrophilic chitosan-polyethylene oxide nanoparticles as protein carriers. J Appl Polym Sci. 63:125-32.

[10] P. Bettencourt, D. Pires, N. Carmo and E. Anes (2010) Application of Confocal Microscopy for Quantification of Intracellular Mycobacteria in Macrophages. Microscopy: Science, Technology, Applications and Education: 614-621. 